CARDIOVASCULAR MEDICINE

\title{
Lack of clopidogrel-CYP3A4 statin interaction in patients with acute coronary syndrome
}

\author{
D Mukheriee, E Kline-Rogers, J Fang, K Munir, K A Eagle
}

Heart 2005;91:23-26. doi: 10.1136/hrt.2004.035014

See end of article for authors' affiliations

....................

Correspondence to: Dr Debabrata Mukherjee, Division of Cardiology, University of Michigan Health System, 1500 E Medical Center Drive, Ann Arbor, Michigan 481030311, USA; dmukheri@ umich.edu

Accepted 24 March 2004
Objective: To assess a clinically significant interaction between cytochrome P450 3A4 (CYP3A4) metabolised statin and clopidogrel.

Design: Prospective single centre cohort study.

Setting: Academic teaching hospital in the USA.

Patients: 1651 patients presenting with acute coronary syndromes between January 1999 and February 2003 were studied. Data on baseline demographics, co-morbidities, and in-hospital management were collected.

Main outcome measure: Association of CYP3A4 metabolised statin and clopidogrel use with in-hospital and six month mortality. The impact of the combined use of a CYP3A4 statin and clopidogrel on six month mortality and major adverse cardiac events was analysed by a risk adjusted logistic regression model.

Results: The odds ratios for six month mortality were: for CYP3A4 statin, $0.43195 \%$ confidence interval (Cl) 0.27 to $0.71, p=0.0009)$; for CYP3A4 statin plus clopidogrel, $0.36(95 \% \mathrm{Cl} 0.23$ to 0.60 , $\mathrm{p}<0.001$ ); for non-CYP3A4 statin, 0.22 (95\% Cl 0.08 to $0.59, \mathrm{p}=0.002$ ); and for non-CYP3A4 statin plus clopidogrel, 0.22 (95\% $\mathrm{Cl} 0.06$ to $0.75, \mathrm{p}=0.016)$.

Conclusions: Use of a combination of a CYP3A4 statin plus clopidogrel was associated with lower six month mortality and morbidity in patients with acute coronary syndromes. There was no significant difference in clinical benefit between a CYP3A4 statin and a non-CYP3A4 statin when used in conjunction with clopidogrel. This suggests that the proposed interaction is probably an ex vivo phenomenon and may not be clinically relevant.
C urrent American College of Cardiology/American Heart Association guidelines recommend co-administration of clopidogrel and statins in patients with acute coronary syndromes (ACS). ${ }^{1}$ Several recent ex vivo platelet function studies have suggested that clopidogrel's effectiveness in inhibiting platelet aggregation is diminished if given together with statins metabolised by cytochrome P450 3A4 (CYP3A4), ${ }^{2-4}$ whereas other studies have shown no interaction. $^{56}$ CYP3A4 metabolised statins include lovastatin, simvastatin, cerivastatin, and atorvastatin. Non-CYP3A4 metabolised statins such as pravastatin, fluvastatin, and rosuvastatin should not interfere with clopidogrel's antiplatelet activity.

We systematically assessed the use of CYP3A4 and nonCYP3A4 statins with concomitant clopidogrel use in patients admitted with ACS at our institution. The independent impact of the statin-clopidogrel combination on six month mortality was ascertained by a risk adjusted logistic regression model.

\section{METHODS \\ Patients}

From 1 January 1999 to 28 February 2003, 1651 patients were admitted to or discharged from inpatient services at our institution with a diagnosis of unstable angina or acute myocardial infarction (MI). All patients were identified by admission or discharge diagnoses and then the charts were reviewed to screen for entry criteria. Inclusion in the study required symptoms consistent with ACS and ECG changes suggestive of ischaemia (ST segment elevation or depression of $\geqslant 1 \mathrm{~mm}, \mathrm{~T}$ wave inversion, or increased cardiac biomarkers). A final diagnosis of MI required increased creatine kinase $\mathrm{MB}(\mathrm{CK}-\mathrm{MB})$ fraction or troponin as described in the American College of Cardiology guidelines. ${ }^{7}$ Reinfarction was defined as recurrent chest pain with new ECG changes (ST elevation of at least $1 \mathrm{~mm}$ in two contiguous leads, new Q waves) or new enzyme increases-that is, a re-increase of CK-MB after reaching a plateau or trough or a $>20 \%$ increase of the previous value of already increased CK-MB. Stroke was identified clinically by a neurological deficit persisting $>24$ hours with or without confirmation by computed tomography or magnetic resonance imaging. The study protocol was approved by the institutional review board at the University of Michigan and informed consent was obtained from all patients.

\section{Data collected}

Clinical, demographic, treatment, and outcome data were abstracted from medical charts by trained abstractors (cardiology fellows and cardiology research nurses). Definitions were based on those recommended by the American College of Cardiology data standards committee. ${ }^{8}$ ECG changes and initial laboratory data were recorded. Data describing patient management covered use of $\beta$ blockers, aspirin, clopidogrel, angiotensin converting enzyme inhibitors, angiotensin receptor blockers, CYP3A4 and non-CYP3A4 statins, and percutaneous coronary interventions or coronary artery bypass grafting. Data on particular statin use was not collected prospectively but rather retrospectively from medical chart records. All other data were collected prospectively for our institutional ACS database. Six month mortality, MI, and stroke data were obtained on $100 \%$ of the patients from a health system record review or telephone call interview.

\section{Statistical analysis}

Baseline characteristics were summarised by the use of frequencies and percentages for categorical factors and mean (SD) for continuous factors. Multivariable logistic regression analysis was performed for six month death and major 


\begin{tabular}{|c|c|c|c|c|}
\hline & \multicolumn{4}{|c|}{ All patients with acute coronary syndromes } \\
\hline & $\begin{array}{l}\text { Clopidogrel }+ \\
\text { no statin }(n=180)\end{array}$ & $\begin{array}{l}\text { Clopidogrel }+ \\
\text { CYP3A4 }(n=561)\end{array}$ & $\begin{array}{l}\text { Clopidogrel + non- } \\
\text { CYP3A4 ( }=91)\end{array}$ & p Value* \\
\hline \multicolumn{5}{|l|}{ Diagnosis } \\
\hline ST elevation MI & $44(24.4 \%)$ & $136(24.2 \%)$ & $15(16.5 \%)$ & 0.10 \\
\hline Non-ST elevation MI & $109(60.6 \%)$ & $335(59.7 \%)$ & $57(62.6 \%)$ & 0.59 \\
\hline Unstable angina & $27(15 \%)$ & $90(16 \%)$ & $19(20.9 \%)$ & 0.25 \\
\hline \multicolumn{5}{|l|}{ Demographics } \\
\hline Age (years) & 64.7 (13.8) & $62.2(12.9)$ & 59.7 (12.5) & 0.08 \\
\hline Male sex & $116(64.4 \%)$ & $390(69.5 \%)$ & $63(69.2 \%)$ & 0.95 \\
\hline Body mass index & $28.4(6.3)$ & $29.3(5.8)$ & $29.1(5.5)$ & 0.76 \\
\hline $\begin{array}{l}\text { Prior angina } \\
\text {. }\end{array}$ & $86(47.8 \%)$ & $265(47.4 \%)$ & $57(63.3 \%)$ & $<0.01$ \\
\hline Prior Ml & $70(39.1 \%)$ & $219(39.3 \%)$ & $38(42.2 \%)$ & 0.60 \\
\hline Prior stroke & $21(11.8 \%)$ & $56(10.1 \%)$ & $8(8.9 \%)$ & 0.73 \\
\hline Prior heart failure & $37(20.6 \%)$ & $65(11.6 \%)$ & $11(12.1 \%)$ & 0.89 \\
\hline Previous coronary intervention & $44(24.6 \%)$ & $183(32.9 \%)$ & $31(34.8 \%)$ & 0.72 \\
\hline Previous bypass surgery & $34(18.9 \%)$ & $122(21.8 \%)$ & $22(24.7 \%)$ & 0.54 \\
\hline Smoking (current or former) & $101(56.1 \%)$ & $358(64.2 \%)$ & $59(65.6 \%)$ & 0.79 \\
\hline Diabetes & $59(32.8 \%)$ & $154(27.5 \%)$ & $26(28.6 \%)$ & 0.82 \\
\hline Hypertension & $149(82.8 \%)$ & $456(81.3 \%)$ & $66(72.5 \%)$ & 0.05 \\
\hline Hyperlipidaemia & $91(50.6 \%)$ & $493(87.9 \%)$ & $72(79.1 \%)$ & 0.02 \\
\hline \multicolumn{5}{|l|}{ Clinical presentation } \\
\hline Positive cardiac biomarkers & $152(84.4 \%)$ & $464(82.7 \%)$ & $72(79.1 \%)$ & 0.40 \\
\hline Serum creatinine $(\mu \mathrm{mol} / \mathrm{l})$ & $124(115)$ & 106 (71) & $97(35)$ & 0.42 \\
\hline \multicolumn{5}{|l|}{ ECG } \\
\hline New ST segment depression & $43(23.9 \%)$ & $134(23.9 \%)$ & 14 (15.4\%) & 0.07 \\
\hline New significant $Q$ wave & $11(6.1 \%)$ & $23(4.1 \%)$ & $3(3.3 \%)$ & 0.71 \\
\hline \multicolumn{5}{|c|}{$\begin{array}{l}\text { Data are mean (SD) or number (\%). } \\
\text { CYP3A4 metabolised statins: lovastatin, simvastatin, cerivastatin, and atorvastatin. Non-CYP3A4 metabolised } \\
\text { statins: pravastatin, fluvastatin, rosuvastatin. } \\
{ }^{*} \text { Comparisons between patients who received CYP3A4 statin versus a non-CYP3A4 statin with clopidogrel. } \\
\text { Categorical variables were compared with } \chi^{2} \text { tests and continuous variables, with } t \text { tests. } \\
\text { MI, myocardial infarction }\end{array}$} \\
\hline
\end{tabular}

adverse cardiac events (MACE; composite of death, MI, and stroke) in patients with ACS treated with the statin plus clopidogrel adjusted for age, sex, positive biomarker, new ST elevation, history of diabetes, renal failure, heart failure, and revascularisation. Both a c-index (a measure of model discrimination) and Hosmer-Lemeshow test (a measure of model calibration) were used to determine the performance of the multivariate models. All data were analysed with SAS version 8.2 (SAS Institute, Cary, North Carolina, USA).

\section{RESULTS}

Table 1 shows the baseline characteristics of the study cohort. Of 1651 patients, 331 patients received neither clopidogrel nor statin, 180 patients received clopidogrel only, 387 used CYP3A4 statin only, 561 received CYP3A4 statin plus clopidogrel, 101 received non-CYP3A4 statin only, and 91 received non-CYP3A4 statin plus clopidogrel. Patients with ACS receiving a CYP3A4 metabolised statin were slightly older, less likely to have a prior history of angina, and more likely to have hypertension and hyperlipidaemia. There were no significant differences in history of prior MI, diabetes, smoking, increased biomarkers, renal function, or clinical presentation between patients stratified by type of statin use.

Table 2 shows cumulative six month clinical event rates for patients treated with a combination of CYP3A4 and nonCYP3A4 statin and clopidogrel. There were no significant differences in clinical outcomes stratified by use of CYP3A4 statin. For example, with atorvastatin as the CYP3A4 statin and pravastatin as the non-CYP3A4 statin, there were no differences in clinical outcome between these agents when used in combination with clopidogrel (fig l).

The multivariate risk adjusted odds ratios for six month death were: for CYP3A4 statin alone, 0.43 (95\% confidence interval (CI) 0.27 to $0.71, \mathrm{p}=0.0009$ ); for clopidogrel alone, 0.48 ( $95 \%$ CI 0.26 to $0.89, p=0.01$ ); for CYP3A4 statin plus clopidogrel, 0.36 (95\% CI 0.23 to $0.60, \mathrm{p}<0.001$ ); for nonCYP3A4 statin, 0.22 (95\% CI 0.08 to $0.59, \mathrm{p}=0.002$ ); and for non-CYP3A4 statin plus clopidogrel, 0.22 (95\% CI 0.06 to $0.75, \mathrm{p}=0.016$ ) (table 3 ). The c-index for this model was 0.80 , suggesting excellent model discrimination. The HosmerLemeshow test statistic was 0.71, suggesting good model calibration and goodness of fit. Table 3 shows the risk

Table 2 Six month outcomes in patients stratified by CYP3A4 metabolised statin use

\begin{tabular}{llllll}
\hline \multicolumn{7}{c}{ All patients with acute coronary syndromes } \\
\cline { 2 - 6 } Outcome & $\begin{array}{l}\text { No clopidogrel }+ \\
\text { no statin }(\mathbf{n = 3 3 1 )}\end{array}$ & $\begin{array}{l}\text { Clopidogrel + no } \\
\text { statin }(\mathbf{n = 1 8 0 )}\end{array}$ & $\begin{array}{l}\text { Clopidogrel }+ \\
\text { CYP3A4 }(\mathbf{n = 5 6 1 )}\end{array}$ & $\begin{array}{l}\text { Clopidogrel + non- } \\
\text { CYP3A4 (n=91) }\end{array}$ & p Value \\
\hline Death & $57(17.2 \%)$ & $18(10 \%)$ & $32(5.7 \%)$ & $3(3.3 \%)$ & 0.24 \\
MI & $14(8.3 \%)$ & $10(8.9 \%)$ & $28(7.6 \%)$ & $9(9.7 \%)$ & 0.39 \\
Stroke & $3(1.1 \%)$ & $2(1.3 \%)$ & $5(1.4 \%)$ & $0(0 \%)$ & 0.70 \\
MACE & $73(22.1 \%)$ & $30(16.7 \%)$ & $64(11.4 \%)$ & $12(13.2 \%)$ & 0.54 \\
\hline
\end{tabular}

*Comparisons between patients who received CYP3A4 statin versus a non-CYP3A4 statin with clopidogrel. MACE, major adverse cardiac outcomes (composite of death, MI, and stroke). 


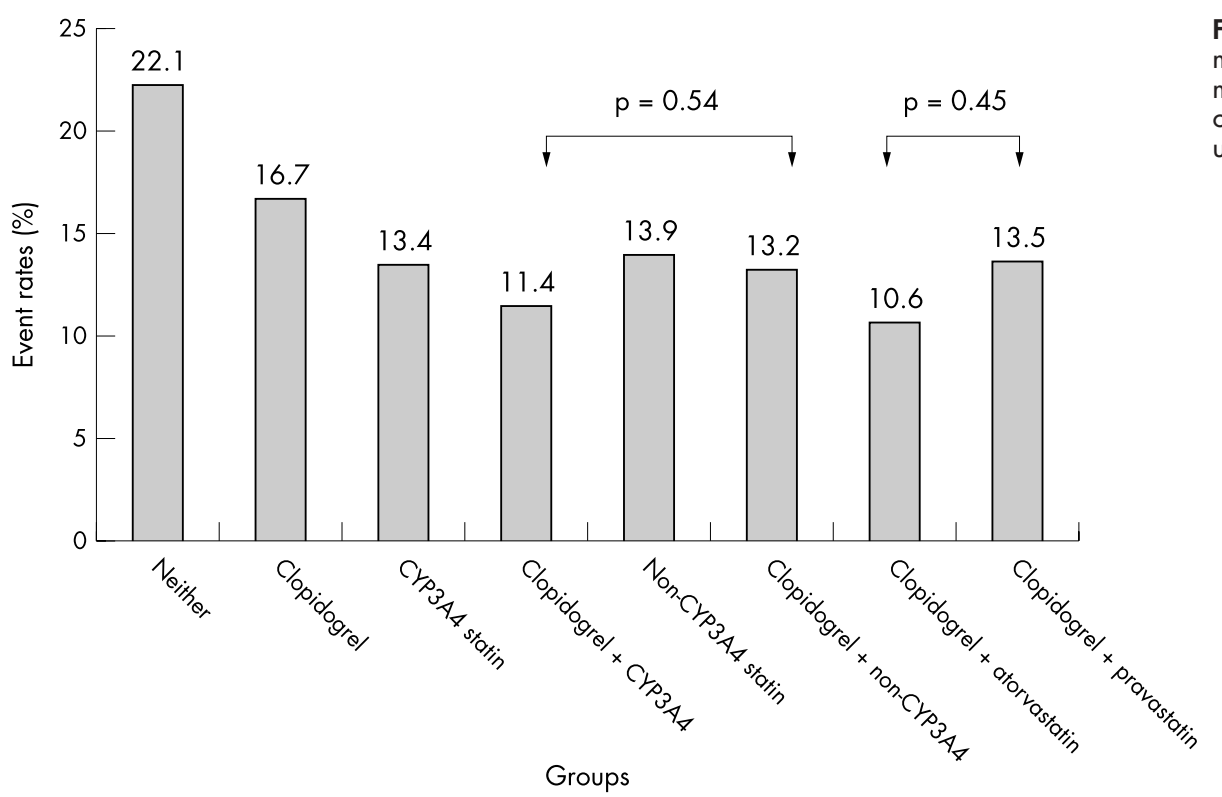

Figure 1 Event rates (death/ myocardial infarction/stroke at six months) in patients stratified by cytochrome P450 3A4 (CYP3A4) statin use and clopidogrel use. adjusted odds ratios for MACE and shows a significant reduction in MACE with the combination of clopidogrel and a CYP3A4 statin (odds ratio 0.53 , 95\% CI 0.36 to 0.79 , $\mathrm{p}=0.001)$.

In a separate model, we compared the association between non-CYP3A4 statin plus clopidogrel and mortality with CYP3A4 statin plus clopidogrel as the reference group. The odds ratio was 0.56 (95\% CI 0.15 to $2.1, \mathrm{p}=0.38$ ) suggesting no significant differences in mortality with type of statin used in conjunction with clopidogrel.

\section{DISCUSSION}

Several recent ex vivo studies have suggested that statins metabolised by CYP3A4 such as atorvastatin may significantly attenuate platelet aggregation inhibition by clopidogrel. ${ }^{2}{ }^{3}$ Such potential drug interactions with clopidogrel may be particularly important to recognise in patients with ACS who may be prescribed clopidogrel for 9-12 months. ${ }^{1}$ We systematically assessed the association of CYP3A4 metabolised statin and clopidogrel use with in-hospital and six month clinical event rates to ascertain whether a clinically significant interaction exits. There was no significant difference in clinical benefit between a CYP3A4 statin and a non-CYP3A4 statin when used in conjunction with clopidogrel. The results are in agreement with the reports by Saw and colleagues, ${ }^{9}$ who found no clinically relevant interaction between CYP3A4 metabolised statin and clopidogrel in a post hoc analysis of a placebo controlled study, and by Wienbergen and colleagues, ${ }^{10}$ who also found no significant difference between atorvastatin and other statins in the clinical outcomes of patients with ACS receiving clopidogrel. The results are also consistent with the report by Muller and colleagues, ${ }^{11}$ who used optical aggregometry for an ex vivo analysis and showed that concomitant use of CYP3A4 statins with clopidogrel did not significantly inhibit antiplatelet activity when clopidogrel was administered at a higher loading dose of $600 \mathrm{mg}$.

In our analysis of patients who received CYP3A4 metabolised statin, clopidogrel was associated with decreased six month mortality and a reduction in MACE. The discordance between the ex vivo data and clinical results may have several potential explanations. One concern is that the sample size in the initial ex vivo studies ${ }^{2}{ }^{3}$ was very small, increasing the likelihood of detecting a spurious association based on biological variability. Moreover, platelet function in ex vivo tests may not adequately reflect the in vivo milieu when agents are administered together. In the only ex vivo analysis that used one of the ideal platelet function measurements, optical aggregometry, ${ }^{12}$ there was no significant interaction. ${ }^{11}$ Another explanation is that the non-platelet aggregation inhibition benefit of clopidogrel in reducing inflammation may result in clinical benefit in these patients. ${ }^{13}$

Table 3 Significant multivariable risk adjusted predictors of six month mortality and MACE after acute coronary syndrome

\begin{tabular}{llccc}
\hline & $\begin{array}{l}\text { Adjusted OR for } \\
\text { death (95\% CI) }\end{array}$ & p Value & $\begin{array}{l}\text { Adjusted OR for } \\
\text { MACE (95\% CI) }\end{array}$ & p Value \\
\hline Age & $1.04(1.02$ to 1.06$)$ & $<0.001$ & $1.03(1.02$ to 1.04$)$ & $<0.01$ \\
Positive biomarker & $1.75(1.11$ to 2.75$)$ & $<0.001$ & $1.76(1.23$ to 2.53$)$ & $<0.01$ \\
Diabetes mellitus & $1.42(0.95$ to 2.09$)$ & 0.08 & $1.29(0.95$ to 1.77$)$ & 0.10 \\
Renal insufficiency & $2.21(1.42$ to 3.44$)$ & $<0.001$ & $2.13(1.46$ to 3.10$)$ & $<0.001$ \\
Heart failure at presentation & $3.88(2.61$ to 5.76$)$ & $<0.001$ & $2.79(1.99$ to 3.84$)$ & $<0.001$ \\
Clopidogrel & $0.48(0.26$ to 0.89$)$ & 0.01 & $0.65(0.39$ to 1.07$)$ & 0.09 \\
CYP3A4 statin & $0.43(0.27$ to 0.71$)$ & 0.009 & $0.55(0.36$ to 0.83$)$ & 0.004 \\
CYP3A4 statin + clopidogrel & $0.36(0.23$ to 0.60$)$ & $<0.001$ & $0.53(0.36$ to 0.79$)$ & 0.001 \\
Non-CYP3A4 statin & $0.22(0.08$ to 0.59$)$ & 0.002 & $0.56(0.28$ to 1.08$)$ & 0.08 \\
Non-CYP3A4 statin + clopidogrel & $0.22(0.06$ to 0.76$)$ & 0.016 & $0.68(0.34$ to 1.67$)$ & 0.28 \\
\hline c-index for the mortality model was $0.80 ;$ c-index for the MACE model was 0.72. & & \\
Cl, confidence interval; OR, odds ratio. & & & &
\end{tabular}


A limitation of our study is its non-randomised nature. We also assumed that patients who were prescribed statins and clopidogrel actually took their medications and we did not confirm compliance. However, we used a multivariate logistic regression analysis to adjust for differences in baseline demographics, co-morbidities, and revascularisation, and the model was robust in its overall ability to predict risk of both death and MACE.

We showed that a combination of a CYP3A4 statin plus clopidogrel was associated with lower six month mortality and MACE in patients with ACS. Although there are some differences in the odds ratios and risk reduction there was no significant difference in clinical benefit between a CYP3A4 statin and a non-CYP3A4 statin when used in conjunction with clopidogrel. The results from this large prospective registry of patients suggest the absence of any significant adverse clinical interaction of these two potent and effective agents in the management of patients with ACS.

\section{Authors' affiliations}

D Mukherjee, E Kline-Rogers, J Fang, K Munir, K A Eagle, University of Michigan, Ann Arbor, Michigan, USA

\section{REFERENCES}

1 Braunwald E, Antman EM, Beasley JW, et al. ACC/AHA guideline update for the management of patients with unstable angina and non-ST-segment elevation myocardial infarction-2002. Circulation 2002;106:1893-900.
2 Lau WC, Waskell LA, Watkins PB, et al. Atorvastatin reduces the ability of clopidogrel to inhibit platelet aggregation: a new drug-drug interaction. Circulation 2003;107:32-7.

3 Neubaver H, Gunesdogan B, Hanefeld C, et al. Lipophilic statins interfere with the inhibitory effects of clopidogrel on platelet function-a flow cytometry study. Eur Heart J 2003;24:1744-9.

4 Bates ER, Mukherjee D, Lau WC. Drug-drug interactions involving antiplatelet agents. Eur Heart J 2003;24:1707-9.

5 Serebruany VL, Malinin Al, Callahan KP, et al. Statins do not affect platelet inhibition with clopidogrel during coronary stenting. Atherosclerosis 2001;159:239-41.

6 Serebruany VL, Steinhubl SR, Hennekens $\mathrm{CH}$. Are antiplatelet effects of clopidogrel inhibited by atorvastatin? A research question formulated but not yet adequately tested. Circulation 2003;107:1568-9.

7 Alpert JS, Thygesen K, Antman E, et al. Myocardial infarction redefined: a consensus document of the joint European Society of Cardiology/American College of Cardiology committee for the redefinition of myocardial infarction. J Am Coll Cardiol 2000;36:959-69.

8 Cannon CP, Battler A, Brindis RG, et al. American College of Cardiology key data elements and definitions for measuring the clinical management and outcomes of patients with acute coronary syndromes. A report of the American College of Cardiology task force on clinical data standards (acute coronary syndromes writing committee). J Am Coll Cardiol 2001;38:2114-30.

9 Saw J, Steinhubl SR, Berger PB, et al. Lack of adverse clopidogrel-atorvastatin clinical interaction from secondary analysis of a randomized, placebocontrolled clopidogrel trial. Circulation 2003;108:921-4.

10 Wienbergen H, Gitt AK, Schiele R, et al. Comparison of clinical benefits of clopidogrel therapy in patients with acute coronary syndromes taking atorvastatin versus other statin therapies. Am J Cardiol 2003;92:285-8.

11 Muller I, Besta F, Schulz C, et al. Effects of statins on platelet inhibition by a high loading dose of clopidogrel. Circulation 2003;108:2195-7.

12 Mukheriee D, Moliterno DJ. Monitoring antiplatelet therapy: what is the best method? Clin Pharmacokinet 2000;39:445-58.

13 Hermann A, Rauch BH, Braun M, et al. Platelet CD40 ligand (CD4OL): subcellular localization, regulation of expression, and inhibition by clopidogrel. Platelets 2001;12:74-82.

\section{IMAGES IN CARDIOLOGY}

\section{Wire artefact-in reverse!}

$\Lambda^{7}$ 75 year old woman with symptomatic hypertrophic cardiomyopathy and a left ventricular outflow tract gradient under stress exceeding $100 \mathrm{~mm} \mathrm{Hg}$ was admitted for alcohol septal ablation. Panel A shows an apparent stenosis in the proximal right coronary artery identified at coronary angiography (left anterior oblique (LAO) $20^{\circ}$, cranial $20^{\circ}$ ) immediately pre-procedure. After the ablation procedure, it was elected to perform percutaneous coronary intervention on this lesion. On passage of a Balance middleweight wire to the distal vessel, however, the "stenosis" resolved (panel B). The lesion reappeared when the wire was withdrawn and resolved again when the wire was passed back down the vessel. It was concluded that this

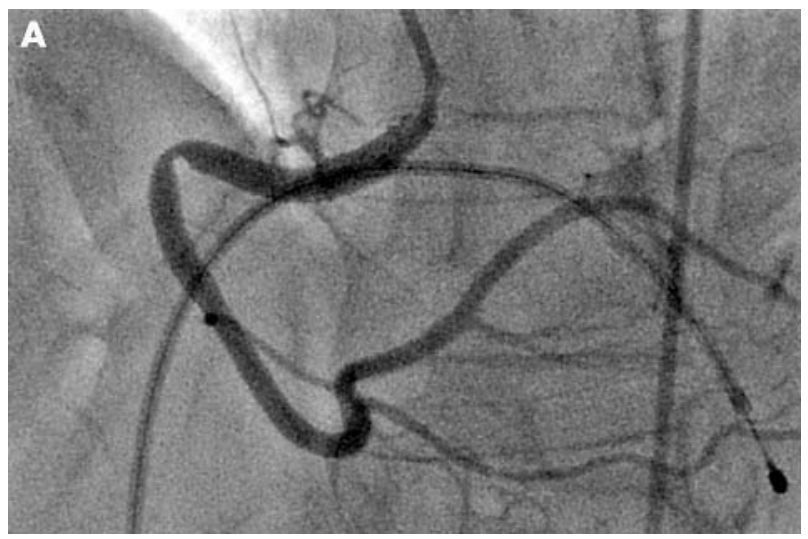

apparent lesion represented a kink in the vessel and no intervention was performed.

It is well recognised that conformational change of a vessel wall by an angioplasty wire can cause appearances suggestive of coronary stenosis or dissection. Such a "wire artefact" occurs most commonly when the wire results in straightening of a tortuous vessel causing rucking of the vessel wall. These images demonstrate quite the reverse-an apparent stenosis that is abolished, rather than caused, by passage of an angioplasty wire.
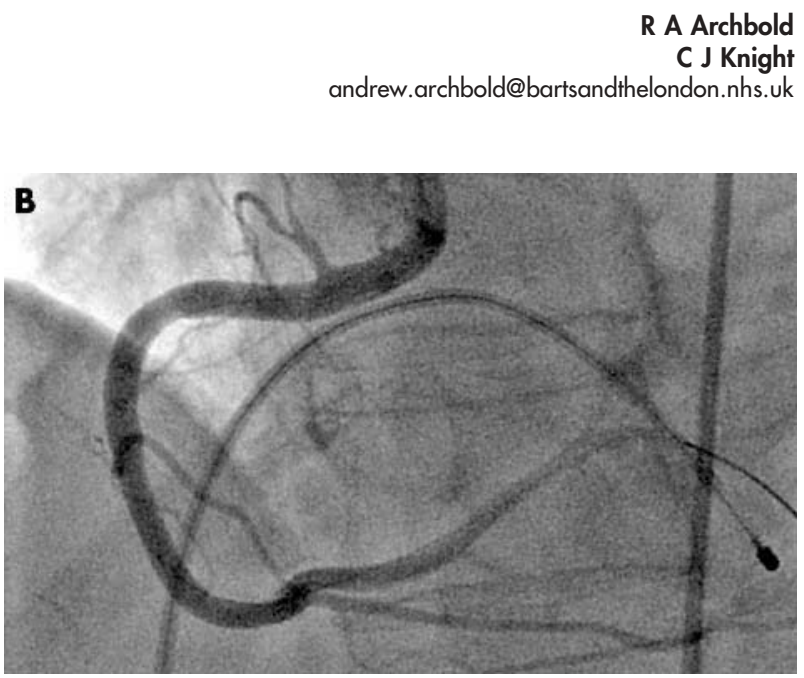